\title{
EXAMINATION OF THE 15-MINUTE LIFE CYCLE PROGRAM OF A CHINESE MEGA CITY: CASE STUDY OF GUANGZHOU
}

\author{
DAILIN ZHOU \\ Guangzhou Urban Planning and Design Survey Research Institute, China
}

\begin{abstract}
This paper empirically explores the configuration of community public facilities under the 15 -minute life cycle program in Guangzhou, which requires community public facilities to be laid out within the 15-minute walking distance. By examining 12 communities with differentiated geographical and demographic features, the paper argues that although the fulfillment rate is acceptable, several problems remain. The 15-minute life cycle program requirement is uniformly imposed, it ignores the demographic structure differences among communities. The needs of seniors and other vulnerable parties are often neglected. Also, for those rural communities with low population density, the uniform configuration requirement leads to the unsustainable operation of the public facilities. Besides that, with the increasing living standards in Guangzhou, like most Chinese cities, new types of public facilities are gaining substantial demand in communities. Those types of public facilities are yet to be included in the program. As such, the 15-minute life cycle program enhances the city's community public facilities provision, but certain optimization strategies should be applied to improve the policy design. Keywords: 15-minute life cycle program, mega city, configuration of public facilities.
\end{abstract}

\section{INTRODUCTION}

The Central Economic Work Conference in China, held in 2015, firstly proposed the concept of the 15-minute community life cycle. It has been rapidly promoted to numerous Chinese cities, especially mega cities and is viewed as the reform to the traditional planning paradigm. The importance of the 15-minute community life cycle is to put residents' needs into core consideration instead of the top-down, rational and resource-allocation thinking.

A typical Chinese mega city like Guangzhou, community-level public facilities are crucial to people's livelihood. Before the program of 15-minute community life cycles, the configuration of public facilities was guided by the regulatory detailed planning, which abode by Guangzhou Urban and Rural Planning Technical Regulation. Although the Regulation gave some variances to the configuration criteria, its intrinsic logic was still allocating the public resources equally and uniformly. This made the Regulation far from meeting people's needs and aroused the necessity of imposing the 15-minute community life cycle program.

The program of the 15-minute community life cycle was launched in 2016, following several planning studies conducted by the Guangzhou Urban Planning Bureau. The contents of the program include the improvements of public facilities, public spaces, residents' commuting pattern and working pattern. The improvement of public facilities was the foci, which aimed to make five types of public facilities laid out within the 15-minute walking distance. The five types of public facilities were education, public health, culture, elderly and sports facilities.

This article intends to refine the 15-minute community life cycle program to be more accordance to people's needs. It firstly examined the existing configuration of communitylevel public facilities and then conducted empirical survey within 12 communities to explore the differentiation between supply and people's demand. Based on the survey, this article concluded with the improvement suggestions to the 15-minute community life cycle program. 
Table 1: The classification of 12 communities.

\begin{tabular}{|l|l|l|}
\hline Classification & Community name & Description \\
\hline \multirow{4}{*}{ Inner city } & San You Li Community & $\begin{array}{l}\text { Residential cluster of government } \\
\text { workers' dependents }\end{array}$ \\
\cline { 2 - 3 } & Jian She Community & Old residential community \\
\hline \multirow{4}{*}{$\begin{array}{l}\text { Newly-developed } \\
\text { city center }\end{array}$} & Blossom Cove & High-grade community \\
\cline { 2 - 3 } $\begin{array}{l}\text { Urban-rural } \\
\text { fringe }\end{array}$ & Tang De Garden & Affordable housing community \\
\cline { 2 - 3 } & Wu Yi New Town & Newly-developed community \\
\hline & HuaNan Country Garden & Large-scaled residential community \\
\cline { 2 - 3 } & Rose Garden & Community located in urban fringe \\
\cline { 2 - 3 } & Longgui Town & $\begin{array}{l}\text { Large-scaled affordable housing } \\
\text { community }\end{array}$ \\
\cline { 2 - 3 } Outer city & He Feng New Town & Rural residential community \\
\hline & Ya Yun New Town & Large-scaled residential community \\
\cline { 2 - 3 } & Luo Chang Community & Rural residential community \\
\cline { 2 - 3 } & Yuan Hua Garden & Community located in urban fringe \\
\hline
\end{tabular}

Table 2: Stratification and names of public facilities.

\begin{tabular}{|l|l|}
\hline Type of public facility & Name \\
\hline \multirow{3}{*}{ Education } & High school, junior high school \\
\cline { 2 - 2 } & Elementary school, kindergarten and daycare center \\
\hline \multirow{2}{*}{ Culture } & Community public health center \\
\cline { 2 - 2 } & Clinic, outpatient department \\
\hline \multirow{2}{*}{ Elderly care facilities } & Community children's palace, community culture center \\
\cline { 2 - 2 } & Reading room, activity room \\
\hline \multirow{3}{*}{ Sports facilities } & Nursing home for the seniors \\
\cline { 2 - 2 } & Senior daycare center, activity room, catering center \\
\hline & Sports center, stadium \\
\cline { 2 - 2 } & $\begin{array}{l}\text { Basketball field, badminton field, fitness path, table } \\
\text { tennis room }\end{array}$ \\
\hline
\end{tabular}

\section{RECENT WORK ON 15-MINUTE COMMUNITY LIFE CYCLE}

The 15-minute community life cycle was a new concept to China, and therefore not many researches had been published in this field. Researches published mainly focused on the strategy to develop the 15-minute community life cycle in the city.

Meng studied different age groups of Shanghai. Based on their daily behaviour features, public facilities demands and walking pattern, she brought up five dimensions to develop the 15-minute community life cycle, which were vitality, integration, precision, accessibility and greenery [1]. Rong and Qiuqing furtherly advanced the research to the implementation of the 15 -minute community life cycle, arguing that the implementation should be relied more on the efficiency of utilizing stock land and needed to arouse the cooperation among government, market and resident [2]-[5].

Wei studied this topic from a different angle. He argued that the delineation of the 15minute community life cycle was very important to the development strategy. He borrowed 
the theory of HOMO-URBANICUS from Professor Hok-Lin Leung, and delineated 500 15minute community life cycles in Wu Han [6].

Based on the current researches, there was still room to contribute to the development strategy of 15-minute community life cycle, especially from the practices of mega cities. Mega cities were faced by huge pressures on public services [7]. More case studies were still needed to expand the toolkit to develop 15-minute community life cycle.

\section{STUDY METHOD}

\subsection{Area of interest}

Guangzhou, the provincial city of Guangdong Province, is located in the Southeast China. It has an area of $7434.4 \mathrm{~km}^{2}$. In 2018 , its resident population has reached 14.9 million with an annual increase of 0.3 million.

Our area of interest is delineated by central eight municipal districts in Guangzhou. The area makes up $41.2 \%$ of the city land area and $82.3 \%$ of the resident population. It is the most densely populated area of the city and therefore it has the highest demand of public facilities.

\subsection{Analytical method}

Based on the purpose mentioned in the introduction, accessibility assessment of communitylevel public facilities was firstly examined. As mentioned in the introduction, the major objective of 15-minute community life cycle program was to lay out five types of public facilities within 15-minute walk distance. The achievement of this objective not only related to the amounts of public facilities, but also the spatial lay out. In order to simulate the actual distance walking from each residential district, we used Application Programming Interface (API) to retrieve the actual road paths and location of all the public facilities from AutoNavi Webservice. AutoNavi is one of the most accurate and comprehensive map service suppliers in China. After achieving the data, we used Geographical Information System, ArcGIS 9.3, to calculate the shortest time obtaining the five types of public facility services for each of the residential district in our area of interest.

In order to examine whether the existing configuration of public facilities met residents' demands, a survey was conducted among 12 sample communities. The criteria of sample community selection was representative and typical. The sample communities were classified based on their geographical locations and residential morphology, which resulted in inner city, newly-developed city center and urban-rural fringe and outer city. Questionnaires conducted afterwards included asking residents' normal trip distance to community-level public facilities and their expectations, demands for public facility types and evaluation towards spatial layout and quality of public facilities.

\subsection{Data collection}

Public facility data was collected in June of 2018. Taking precision, obtaining cost and coverage into consideration, the data of public facilities was collected in the format of Points of Interest (POI), including name, type, address and spatial coordination of each facility. The initial amount of public facilities collected was 93,988. After prepossessing to eliminate the duplicated data, the final amount of public facilities was 78,323.

The sample community survey was conducted during April to May 2018. To guarantee the representations of interviewees, each of the community was visited twice, one in weekday and one in weekend and the chosen interviewees varied from age, gender, occupation. The 


\begin{tabular}{|c|c|c|c|c|c|}
\hline \multirow{5}{*}{ 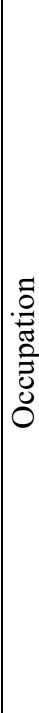 } & $\begin{array}{l}\infty \\
\stackrel{0}{0} \\
\stackrel{5}{0}\end{array}$ & $\begin{array}{l}0 \\
\infty \\
\infty \\
\dot{m}\end{array}$ & $\begin{array}{l}0 \\
\infty \\
n^{0}\end{array}$ & $\begin{array}{l}\stackrel{0}{n} \\
\text { ले }\end{array}$ & 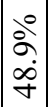 \\
\hline & 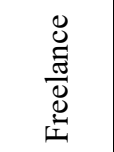 & $\begin{array}{l}\stackrel{0}{ } \\
\delta_{0}\end{array}$ & 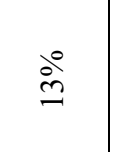 & ठ̊ & ठ̊ㅇ \\
\hline & 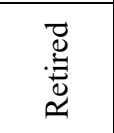 & $\begin{array}{l}\stackrel{0}{ } \\
\infty \\
\text { సે }\end{array}$ & $\begin{array}{l}\stackrel{0}{\circ} \\
\dot{+}\end{array}$ & $\begin{array}{l}\stackrel{\circ}{\sigma} \\
\dot{+}\end{array}$ & $\begin{array}{l}0 \\
\grave{\infty} \\
\infty\end{array}$ \\
\hline & 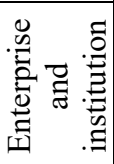 & $\begin{array}{l}\text { ते } \\
\text { ते } \\
\text { ñ. }\end{array}$ & $\frac{\partial^{\circ}}{\gamma}$ & $\stackrel{\stackrel{0}{i}}{\stackrel{y}{y}}$ & 吕 \\
\hline & 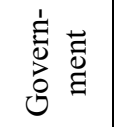 & $\begin{array}{c}\stackrel{0}{ } \\
\text { ले }\end{array}$ & $\begin{array}{l}\stackrel{0}{ } \\
\dot{+}\end{array}$ & लें & ते \\
\hline \multirow{3}{*}{ 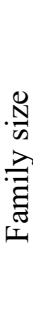 } & $\frac{8}{8} n$ & $\begin{array}{l}\stackrel{0}{0} \\
\stackrel{\vec{j}}{ }\end{array}$ & 仓ें & 犬े & $\begin{array}{l}0 \\
\dot{0} \\
\dot{\Xi} \\
\end{array}$ \\
\hline & $\stackrel{\sim}{\sim}$ & $\frac{\partial^{\circ}}{2 \dot{0}}$ & $\begin{array}{l}\stackrel{0}{0} \\
\dot{+} \\
\dot{0}\end{array}$ & $\begin{array}{l}\text { लें } \\
\text { लें }\end{array}$ & \begin{tabular}{|l|}
0 \\
$b$ \\
$\infty$ \\
0 \\
0
\end{tabular} \\
\hline & $\dot{\bar{\theta}}_{\tilde{g}} \sim$ & $\begin{array}{l}\stackrel{0}{0} \\
\grave{a} \\
\end{array}$ & $\begin{array}{l}\stackrel{0}{2} \\
\ddot{n}\end{array}$ & $\begin{array}{l}\stackrel{0}{i} \\
\stackrel{0}{\infty}\end{array}$ & $\begin{array}{l}0 \\
\infty \\
0 \\
2 \\
0\end{array}$ \\
\hline \multirow{3}{*}{$\stackrel{0}{\stackrel{0}{<}}$} & 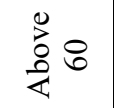 & 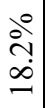 & 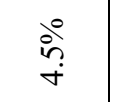 & $\begin{array}{l}\infty \\
\infty \\
\infty \\
\infty\end{array}$ & $\frac{0}{\infty}$ \\
\hline & $\begin{array}{l}8 \\
0 \\
0 \\
\text { i }\end{array}$ & $\begin{array}{l}\stackrel{0}{\grave{n}} \\
\stackrel{2}{2}\end{array}$ & $\begin{array}{l}\stackrel{2}{i} \\
\ddot{\infty}\end{array}$ & $\begin{array}{l}\stackrel{0}{i} \\
\infty \\
\infty\end{array}$ & $\begin{array}{l}\stackrel{2}{i} \\
2 \\
i\end{array}$ \\
\hline & $\frac{\bar{d}}{\tilde{g}} \stackrel{\sim}{\sigma}$ & i̊ & $\begin{array}{l}\stackrel{0}{ } \\
\infty \\
a\end{array}$ & ìn & 仓̊̀ \\
\hline \multirow{2}{*}{ 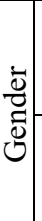 } & $\Sigma$ & 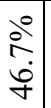 & 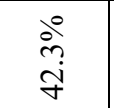 & $\begin{array}{l}\stackrel{2}{a} \\
\dot{\dot{b}}\end{array}$ & $\begin{array}{l}\text { Do } \\
i n \\
\dot{0} \\
+\end{array}$ \\
\hline & 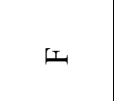 & $\begin{array}{l}\stackrel{0}{ } \\
\text { ñ. } \\
\tilde{n}\end{array}$ & $\frac{\stackrel{\circ}{i}}{i n}$ & iे & $\begin{array}{l}0 \\
i n \\
n \\
n\end{array}$ \\
\hline & 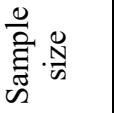 & $\overrightarrow{\mathrm{d}}$ & $\underset{\sim}{\infty}$ & ঐ) & 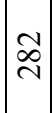 \\
\hline & & 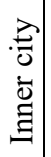 & 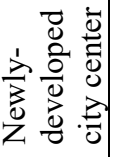 & 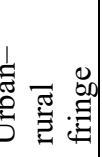 & 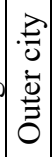 \\
\hline
\end{tabular}


amount of questionnaires collected was 1,090 out of 1,150 distributed. Therefore the rate of questionnaire reclamation was $94.8 \%$, in the acceptable confidence level.

\section{ACCESSIBILITY ASSESSMENT ON EXISTING PUBLIC FACILITIES}

\subsection{Overall assessment results}

Based on our assessment methods, within the study area, the overall fulfillment rate (the proportion of residential districts which were able to fulfill the goal of 15-minute community life cycle from all the residential districts assessed) was $68.3 \%$. The result was modest, showing that there was still a long way to achieve our ideal situation.

The assessment also indicated a large variance among the four classified areas. The fulfillment rates of residential districts in inner city, newly-developed city center, urban-rural fringe and outer city were $88.2 \%, 73.8 \%, 50.3 \%$ and $45.1 \%$ respectively.

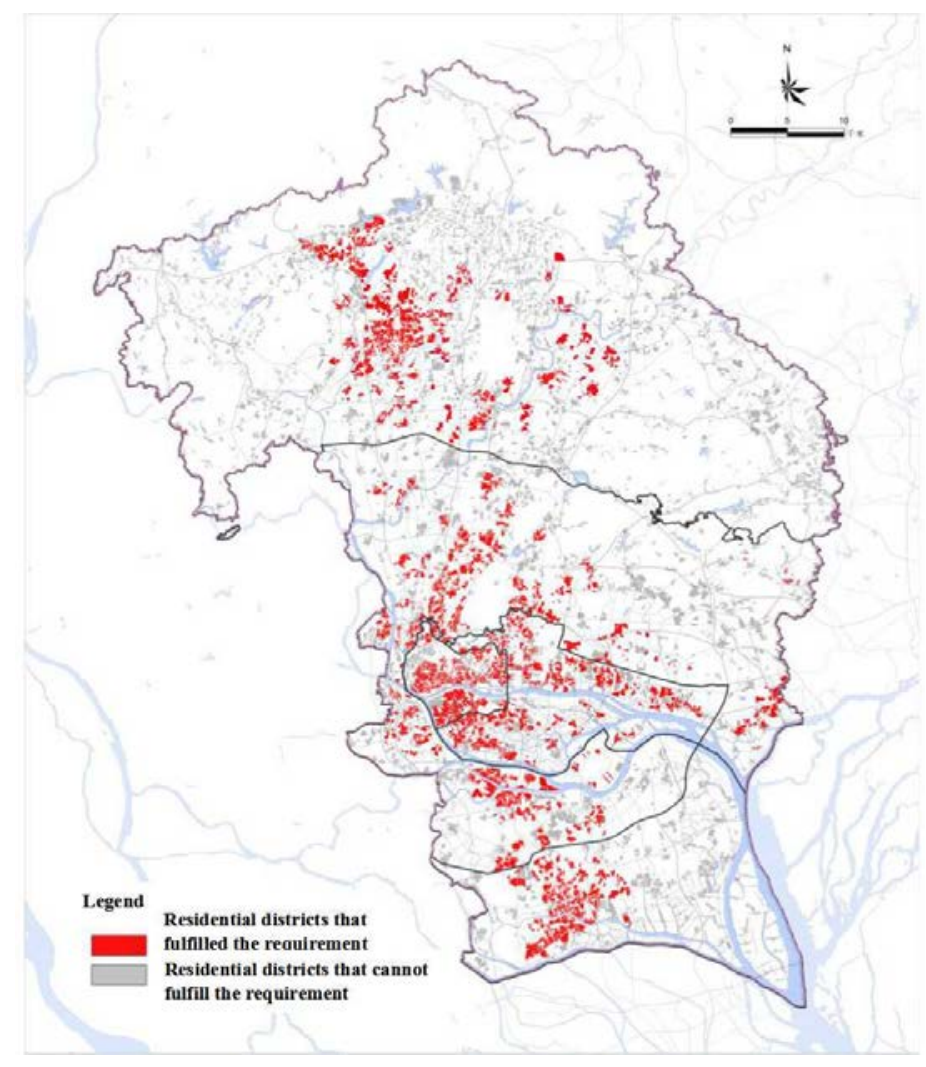

Figure 1: The accessibility result of the assessment.

We further found that there was a high correlation between the fulfillment rate and the population density. 20,000 person $/ \mathrm{km}^{2}$ was a population density breaking point in the case of Guangzhou. Beyond that breaking point, we found that all the residential districts could fulfill the requirement of 15-minute community life cycle. But for those under the breaking point, the fulfillment rate varied substantially. 
Built on our findings, we concluded that to fulfill the goal of 15-minute community life cycle, not only the configuration of public facilities matter, but also the cluster of people. A moderate high population density should be encouraged in order to sustain the development of 15-minute community life. For the residential districts that have already achieved the requirement, more refined objectives should be given to further improve the quality, not just the quantity only. But for those who haven't, especially those with low population density, increasing the amount of public facilities and encouraging small-sized public facilities were still the top priority. It is also worthwhile to pay attention to the extreme-low population density area, which was below 1,000 person $/ \mathrm{km}^{2}$ in the case of Guangzhou. Their 15 -minute community life cycle development strategy should be separately discussed since that even if they fulfil the requirements by adding more facilities, the maintenance of the facilities could be a big problem.

\subsection{Assessment results for different public facilities}

By furtherly assessing the accessibility among different public facilities, we came into the conclusion that accessibility level also varied among different public facilities. Education, public health and sports facilities were the best three public facilities fulfilling the 15-minute community life cycle requirements. However, culture and elder care facilities were far from it, especially supplementary level culture facilities and basic level elder care facilities.

It was interesting to find that the accessibility of basic level public facilities were worse than the supplementary level facilities, which to the common sense, should be the other way around. It indicated that the development of 15-minute community life cycle should pay more attention to the configuration of basic level public facilities, which were usually smaller, scatter and more difficult to manage.

In different areas, the accessibility of different public facilities also differentiated. For example, culture and basic level elder care facilities in inner city were most needed to be improved. But in urban-rural fringe area, culture, sports, elder care facilities and supplementary level public health facilities were all far from satisfaction.

\section{DEMANDS FROM THE COMMUNITY RESIDENTS}

Compared to the accessibility assessment, most of the residents thought that the public facilities were quite accessible and convenient, giving positive comments on the 15 -minute community life cycle program. However, the unity of the program was challenged most.

\subsection{Expectations on trip distance to public facilities}

The average trip distance to public facilities measured in time was 23.1 minutes. The percentage of residents agreeing that they can reach public facilities in 15 minutes was $73.2 \%$, which was better than accessibility assessment result.

However, the gap of accessibility among different areas still remained. The percentage in inner city, newly-developed city center, urban-rural fringe and outer city was $75.4 \%, 74.3 \%$, $62.1 \%$ and $50.3 \%$ respectively. It is important to note that around $30 \%$ and $40 \%$ of the interviewees mentioned that they were not able to reach public facilities by feet, especially those in low density rural communities. This echoed the accessibility assessment on putting special strategies to improve the accessibility in low density communities.

- The place I live is very inconvenient. If I or my daughter is ill, I have to drive to the only hospital in the town center. Junior high school is far away too.

Mr Liu, 45, resident in He Feng New Town 
Also, we found that age was a very important factor in time expectation to public facilities. For the interviewees above age 70, they were far more sensitive to the time needed to reach the public facilities. Their community life-cycle radius was usually 5-10 minutes. This indicated the necessity to further break down the 15-minute life cycle.

- $\quad 15$-minute is too long for me. Every day I go to the community garden to meet my friends. I hope the public facilities which I often use can all be laid out around the garden. 10-minute life cycle is ideal for me.

Mrs Zhang, 71, resident in Jian She Community

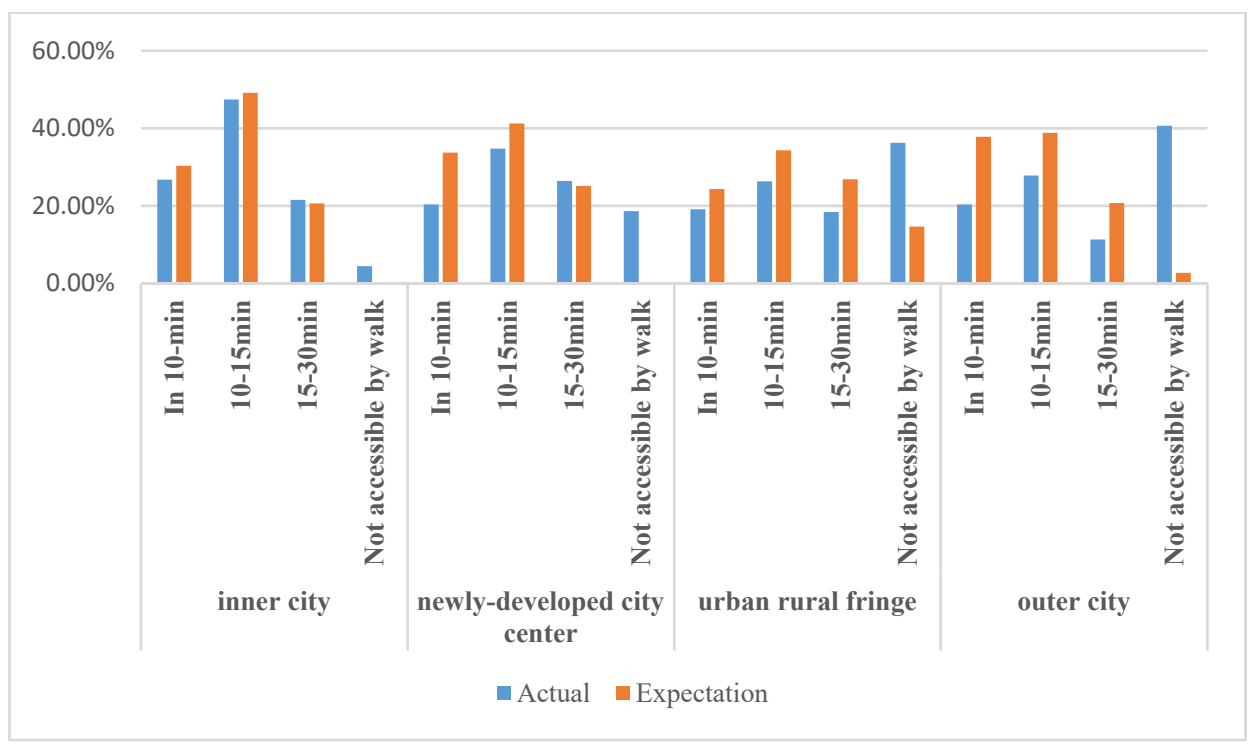

Figure 2: Results of expectations on trip distance to public facilities.

\subsection{Demands of public facility types}

When asked what the most needed public facilities were (existing or non-existing) in 15minute community life cycle, residents in four different areas showed some similarities. Interestingly, the similarities came in two pairs, inner city and newly-developed city center, urban-rural fringe and outer city. For the first pair, the top three needed public facilities were more lean to supplementary level facilities, which were community children's palace, community culture center and pocket garden. For the second pair, public facility demands were still basic. Clinic, senior daycare center and fitness path were the top three most needed public facilities. We can draw a conclusion that the demands of public facilities in urbanrural fringe and outer city were more pressing, since the basic level public facilities were not very complete.

On the other hand, we found that new types of public facilities were gaining substantial demands in communities. For example, the children's daycare center for under the age of 3 , canteen and teaching school for elderly people were mentioned numerous times during the survey. These types of public facilities were left out in 15-minute community life cycle program and thus no clear planning requirements for them. This led to complaints about their site selection, size and other issues. 
- We are having the second-child policy now, but the public facility configuration is too fallen behind. To save money, the daycare centers for small children are usually operated upstairs, making no room for outdoor activities. The children can only play on the pedestrian roads. So sad!

Mrs Zhu, 31, resident in Blossom Cove

Inner City

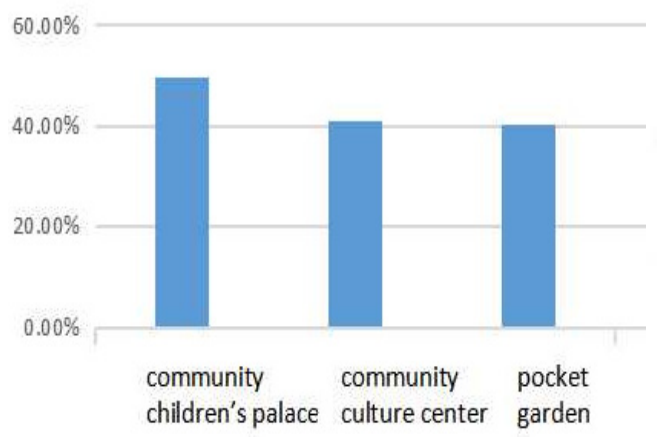

Outer City

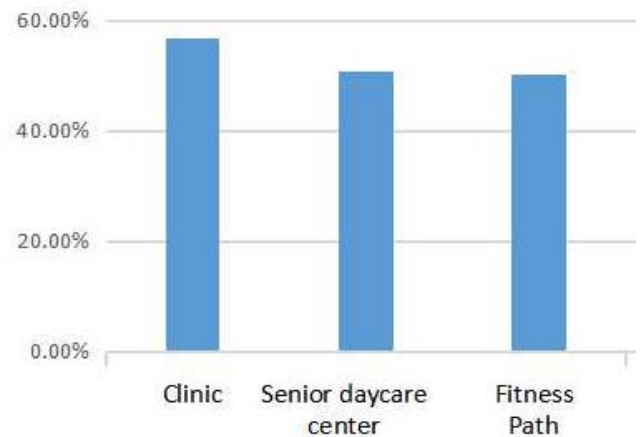

Figure 3: Comparison of demand in public facilities between inner city and outer city.

\subsection{Performance evaluation}

In accordance with accessibility assessment, inner city area had the highest performance satisfaction, and outer city had the lowest, indicating that accessibility might be an important factor deciding the satisfaction degree of public facilities. However, in urban-rural fringe area, we noticed that clinics, although their accessibility were satisfactory $(63.2 \%)$, comments like "not reliable" were made by residents. For those public facilities that have close ties to people's livelihood, high input from the government is still badly in need.

- The doctors in the clinic near where I live are not very good. Last time I went there, I was diagnosed a disease that was not existent at all. It cost me a lot of time and money to figure it out. I won't go there anymore.

Mr Feng, 26, resident in Longgui Town

Performance evaluation results also varied among different types of public facilities. We found that usually education and public health facilities were rated better than culture, elder care and sports facilities. We inferred that since education and public health facilities were more critical to public interest and not-for-profit, they were under stricter supervision from the government. But for culture and sports facilities, they were usually co-operated by private and public organizations, their supervision were less clear. Based on Maslow's hierarchy of needs, culture and sports facilities can be crucial to social acceptance and sense of belonging to the community. With the increasing living standards for cities like Guangzhou, we should start to focus more on the supplementary public facilities.

- The types of books are not very interesting. I would like more novels in the reading room instead of business management books.

Mr Shen, 29, resident in Tang De Garden 


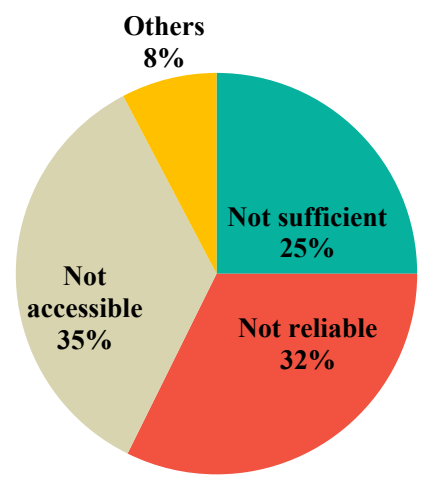

Figure 4: Comments about clinics made by residents in urban-rural fringe area.

\section{CONCLUSIONS AND DISCUSSIONS}

Based on our assessment, the 15-minute community life cycle program had a fair result, with around $64.3 \%$ of the residential districts meeting the requirements of the program. However, the unity of the program was challenged most during our survey in the sample communities. In light of this, diversity is the issue 15 -minute community life cycle program should address most.

\subsection{Spatial differentiation should be respected in the policy design}

Classifying our study area into four districts, spatial differentiation in public facility accessibility, demands of public facility types and preferential rates varied substantially. With high population density, inner city and newly developed city center enjoyed better public accessibility and higher satisfaction about their performance. Their residents' demands also shifted to more supplementary and spiritual types of public facilities. In contrast to the above areas, urban-rural fringe and outer city areas needed more regulations and monitored. With the population density under 20,000 person $/ \mathrm{km}^{2}$, public facilities cannot sufficiently provide without regulation of the government. Therefore, those areas should focus more on the provision of basic types of public facilities. For some areas with extreme low population density, the way to provide should be deliberately considered.

\subsection{Demands of the seniors and other vulnerable parties should be carefully considered}

During our survey, we found that seniors above age 70 were very time sensitive to the trip distance to public facilities. 15 minute walking distance is not suitable for different age groups. In the areas with higher elderly population, such as the inner city, a 5-minute or 10minute life cycle should be broken down from the 15-minute community life cycle program.

With the change in demographic structure of the society, new types of public facilities also need to be considered. As China is implementing the second child policy, children's daycare center for under the age of 3 is badly needed in 15-minute community life cycle. Without appropriate planning regulations will decrease the quality of community life cycle. 
6.3 Other demographic features are also important in the policy design

As other mega cities in China, demographic structures are becoming increasingly diversified. Other than age, communities with transient population or international immigrants, could have different patterns in their daily habit and demands of public facility types. Therefore, their needs for 15-minute community life cycle program also worth deeply scrutinized. To do this, we can make the 15-minute community life cycle program more inclusive and increase the welfare of our communities.

\section{ACKNOWLEDGEMENTS}

The project team members have made important contributions to this paper, who are $\mathrm{Mrs} \mathrm{Hu}$ Jiapei, Mrs Wu Lijuan, Mr Wang Ye and Mr Teng.

\section{REFERENCES}

[1] Meng, L., The planning strategies of a 15-minute community life circle based on behaviors of residents. Urban Planning Forum, 2017(1), pp. 111-118, 2017.

[2] Rong, C., Planning and practice of a 15-minute community living circle in Shanghai guided by promoting implementation. Shanghai Urban Planning Review, 2018(2), pp. 84-88, 2018.

[3] Dongfan, X., Qiuqing, W., Minqing, Z. \& Yinan, Z., Exploration of planning and construction of community living circle in shanghai facing 2040. Shanghai Urban Planning Review, 2017(4), pp. 65-69, 2017.

[4] Rong, C., Spatial governance measures of 15-minute life circle with a general perspective. Planners, 2015(5), pp. 115-121, 2015.

[5] Qiuqing, W., The exploration on the dynamic programming of community in megacities from the living circle perspective. Shanghai Urban Planning Review, 2015(4), pp. 1319, 2015.

[6] Wei, W., Mengyao, H. \& Bo, X., Demand-supply matching oriented 15-minute community life circle demarcation and spatial optimization. Planners, 2019(4), pp. 1117, 2019.

[7] Globe Scan \& MRC McLean Hazel, Megacity Challenge A Stakeholder Perspective, Siemens AG Corporate Communications (CC), p. 6, 2007. 